\title{
Comparison of linear control algorithms for a class of nonlinear mechanical actuators
}

\author{
Clara M. IONESCU and Robin DE KEYSER \\ Ghent University, Department of Electrical energy, Systems and Automation \\ Technologiepark 914, 9052 Zwijnaarde, Belgium Email: claramihaela.ionescu@UGent.be
}

\begin{abstract}
This paper presents a comparison between various control strategies for a class of mechanical actuators common in heavy-duty industry. Typical actuator components are hydraulic or pneumatic elements with static nonlinearities, which are commonly referred to as Hammerstein systems. Such static nonlinearities may vary in time as a function of the load and hence classical inverse-model based control strategies may deliver sub-optimal performance. This paper investigates the ability of classical linear control strategies as lead, P, PI and PID control to satisfy tolerance interval for position error values, overshoot and settling time specifications. Due to the presence of static nonlinearity, control effort is also evaluated in terms of zero crossing frequency (up-down or left-right movement). Simulation and experimental data from a lab setup suggest that advanced control strategies may be needed to improve global performance parameters.
\end{abstract}

Index Terms-Hammerstein systems, static nonlinearity, PID control, mechanical actuator

\section{INTRODUCTION}

A class of mechanical systems with nonlinear characteristics are found in automotive industry. To meet future requirements on automotive systems in terms of increased engine power, while reducing fuel consumption and pollutant emissions, new camless engines are required [1]. One of the most common solutions is an independent valve actuator system able to realize Variable Valve Actuation (VVA) operations. To this aim, a promising solution relies on Electromechanical Valve Actuators (EMVA) technology, where an electronic control system commands each valve properly at every engine speed [2]. The effectiveness of the EMVA system depends on the performances of the control strategies necessary to guarantee precise valve closing/opening. The control must account for the behaviour of the system which is strongly affected by many nonlinearities such as friction, motion constraints, delays, etc.

Another class of position controlled mechanical actuators are encountered in agricultural machines. Of the many agricultural machines available in today's mechatronic applications, the harvesting machines are the most difficult to automatize due to the presence of nonlinear dynamics, time delay, uncertainties in model parameters and interaction with the environment (i.e. crop dynamic environment) [3], [4]. A special class of harvesting machines are those composed of tilting and rotating mechanisms for crop harvesting and/or processing. Main types are: i) grain harvesting (tilting the header for distance to the ground regulation) and ii) forage harvesters (rotating the spout for angle of crop flow to a reservoir), both for position control applications.
Challenges for control are numerous, since these complex mechanical systems are inter-coupled with sub-systems delivering pressure, flow or current and which in turn depend on the net power available in the machine. The required power may change in time due to environmental conditions (slope, crop density, speed of harvesting, etc) [5]. Modelling based on physical/mechanical/electrical principles is nearly impossible and thus black box modelling is usually performed via identification techniques to obtain a simplified model of the sub-system and design control strategies.

In this paper we investigate the application of linear control systems to position control of a mechanical system with inputoutput slew rates, saturation, varying time delay and static nonlinearity. The system is challenging for control since it has varying time delay combined with very fast dynamics in the actuators (motor); hence a time delay with an integrator.

The paper is organized as follows: the next section presents the various parts of the system to be controlled. Section III introduces the linear control strategies and the corresponding controller design parameters. Section IV presents the result in simulation and in comparison on real experimental data. A conclusion section summarizes the main outcome of this work and points out towards further improvement in the control strategy to fulfil the required specifications.

\section{A CLASS OF HAMMERSTEIN MODELS}

Actuator solutions in heavy-duty industrial applications where torque and force may be required at high speed and in varying time conditions are usually hydraulic-based elements. The hydraulic circuit is mostly controlled by means of proportional servo valve (PSV) which may be pressure dependent. In order to make things easier from point of view of dynamic compensation of nonlinear effects, one may attempt to make this PSV pressure independent by using a pressure compensated flow restriction valve. The flow usually feeds a gearotor motor transforming it into rotation movement. The modelling difficulties for control purposes are the large range of operating points, fast system dynamics and the lack of sensors in such subsystems.

Depending on the load and its corresponding force a safety mechanism based on spring-damper elements prevents the motor from overload. In the practical operating range, this mechanism will absorb most of the influence of the inertia and some backlash in the gearing. As such, friction is the 
main origin of torque on the engine and dynamical influences are difficult to model.

In the literature, a number of modelling strategies for hydraulic systems are proposed. The most common way to model the kind of systems discussed in this thesis is using greybox non-linear models [6], [7], [8], based on basic hydraulic principles described in [9] or via bondgraphs [10]. The systems discussed in these papers are academic setups with a high number of precise sensors (pressure sensors, spool position sensors) required for fast and precise control, e.g. in active suspension systems. This makes identification of the separate subsystems and system control a lot easier compared to reallife situations. An overview of linear and nonlinear modelling techniques can be found in [11], [12]. Here, the comparison between linear models, neural networks and wavelet networks and their different approaches is being made. As the latter two methods are computational heavy, no good results were achieved due to the lack of sensor precision, and basic (nonlinear) models are required for the subsequently discussed control strategies, one may consider a simplified model.

A simple representation of such mechanical systems is the Hammerstein model structure. For this, the static nonlinearity needs to be defined. The main non-linear behaviour of the system is exerted in the hydraulic part. The dynamics are rather fast, hence they can be ignored in this part of the subsystem. Typical non-linearities which may be found in such mechanical systems are of the following forms:

- Dead zone: The spring force present in the servo valve is the main source of dead zone. The origin lays in the excess spring force which needs to be overcome before the spool moves in order to prevent unwanted spool movement.

- Saturation in the hydraulics: As pressure and orifice diameters are limited, the flow is limited as well. This results in a maximum angular velocity of the system, i.e. can be represented as a slew rate.

- Mechanical limits: This is the limit preventing the excess rotation for safety measures (to avoid hitting other subsystems operating at same time and within same space).

Figure 1 depicts the static characteristic present in our system as available from experimental data. The various colours depict the changes of the operating range, as a result of different load conditions (e.g speed and type of product).

Another source of difficulty in closed loop control operation is the presence of time-delays. The delay mainly depends on the hydraulic system, more exactly on the spool position and pressure build-up in the tubing in the system. Because of the lack of precision in the angle sensor, it is very difficult to define the transition between the delay and the beginning of the dynamics.

Delay estimation techniques are numerous and vary from simple signal processing cross-correlation algorithms [13] to more complex time varying window estimation algorithms [14], [15]. Delay compensators can be used but they require complex control architectures [16]. In our situation, we as-

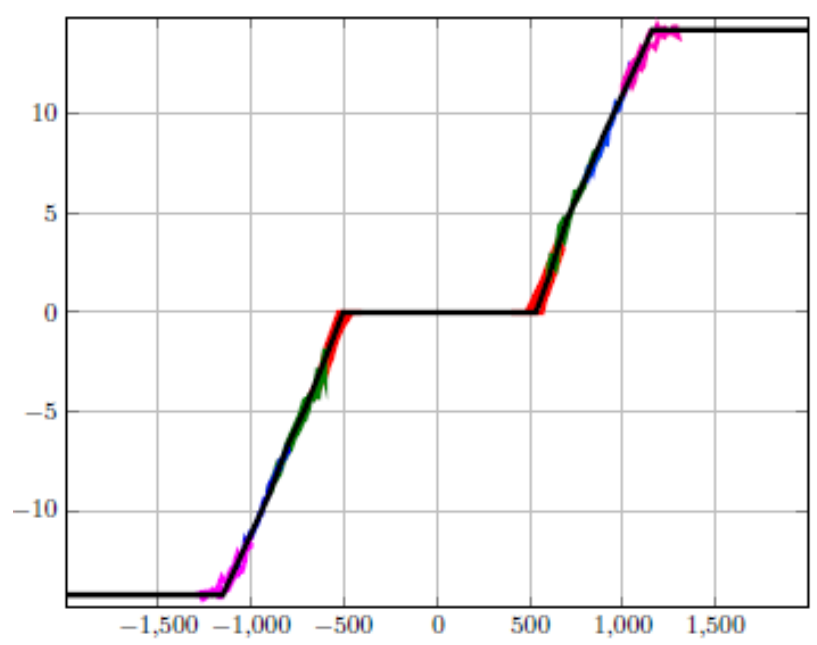

Fig. 1. Static characteristic of the nonlinearity present in the system; notice that the slope may vary depending on the operating conditions, i.e. crop speed and volume per time unit. Arbitrary units: X-axis current and Y-axis flow.

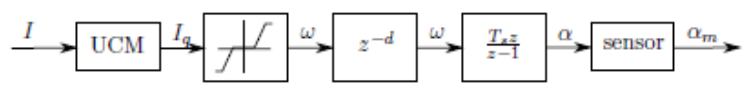

Fig. 2. Block scheme of the open loop system.

sumed the delay is fixed to a certain value and used it directly in the controller design, assuming a robust controller.

If one integrates the above into a Hammerstein-model, one obtains a static non-linear characteristic with linear dynamics, with time delay, as schematically depicted in figure 2 . In this figure, the universal controller module (UCM) and the sensor block represent the quantization in the system. We assume that the angle sensor has a limited precision, i.e. introduces an output quantization of $0.75^{\circ}$. As the current is sent through the controller area network (CAN)-bus to the UCM, also an input quantization of $10 \mathrm{~mA}$ is present in the system.

To summarize, the dynamic part of the following blocks after the static nonlinearity block can thus be represented by the following transfer function:

$$
\frac{\alpha(s)}{\omega(s)}=\exp ^{-0.009 s} \frac{1}{s}
$$

from angular velocity ( $\mathrm{rad} / \mathrm{s})$ to angle position (degrees).

\section{LINEAR CONTROL STRATEGIES}

The default controller available for testing closed loop position control is a $\mathrm{P}$ controller with gain scheduling. Gain scheduling algorithms may prove quite effective when used with advanced control strategies [17]; however, due to the increased numerical complexity these cannot be used in this application. The $\mathrm{P}$ controller was tuned using trial an error. The controller output is converted to a current value using the inverted non-linear characteristic, as in figure 3. 


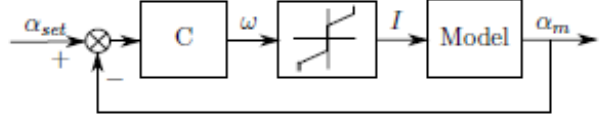

Fig. 3. Default closed loop control scheme for position control.

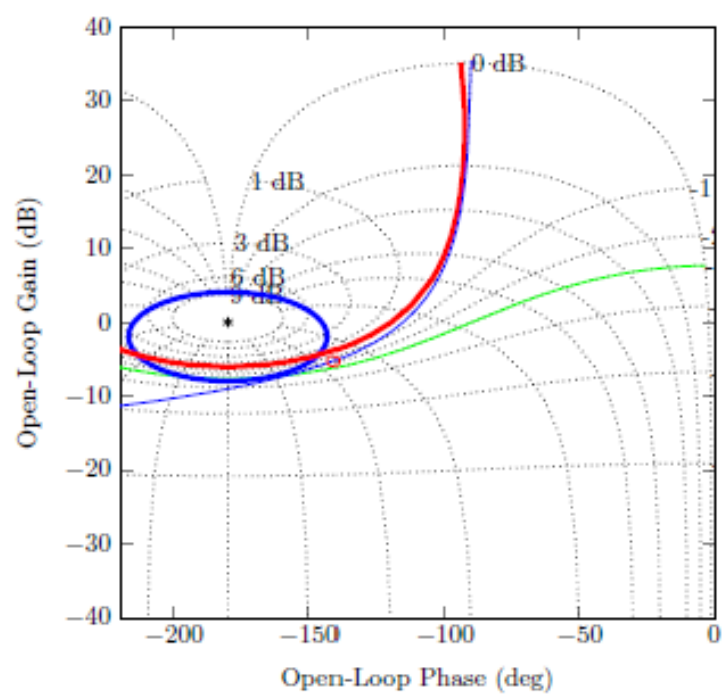

Fig. 4. A snapshot of the FRTool CAD package for controller design available in our laboratory.

We will assume the same configuration for the subsequent control strategies and focus on the design of the C-block only. Lead, PI and PID controllers are taken in consideration, due to their simplicity and hence high implementation ability in modular communication systems available in industry. For tuning the controllers, we make use of the computer aided design tool developed in our lab and under Matlab use: FRTool [18]. A snapshot of the interface is given in figure 4 where the process and controller frequency response is viewed in the Nichols chart along with specifications such as robustness, overshoot, settling time, etc. FRTool Nichols chart of the lead controller. Robustness $>60 \%$ if outside thick blue ellipse, overshoot $<5 \%$ if outside thick red curve, settling time $<0.5 \mathrm{~s}$ if small red circle lays above green $-3 \mathrm{~dB}$ line.

The lead controller earns its name from the fact that the phase shift of this controller will be positive for low frequent input signals, as its zero lays closer to the origin $(\mathrm{s}=-20)$ than the pole $(s=-30)$. In order to get the settling time lower than $0.5 \mathrm{~s}$, the gain of the controller is chosen to be $\mathrm{K}=9$ :

$$
C(q)=K \frac{s+z}{s+p}
$$

with $z$ the zero and $p$ the pole of the controller.

The main disadvantage of the lead controller is the lack of an integrator, as a double integrating closed loop system is required for the elimination of SS type-II errors. Hence, a pure

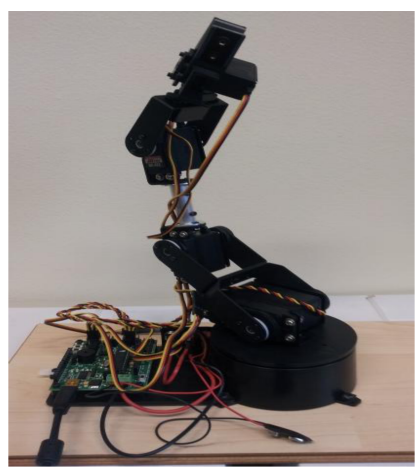

Fig. 5. A picture of the lab setup used to test the control strategies.

integrator is introduced in the control loop. Using FRTool, a PI controller for low overshoot is designed. The formula used for implementing this controller is

$$
C(s)=K \frac{s+K_{i}}{s}
$$

Trade off between fast performance and overshoot results in the following values for $\mathrm{K}=5.41$ and $K_{i}=0.235$.

Finally, a PID controller was designed using FRTool. Two real zeros were chosen, $s_{1}=-0.3713$ and $s_{2}=-33.68$. Using following notation for the controller, with $\mathrm{K}=7.491, K_{d}=$ 0.029 and $K_{i}=0.367$ :

$$
C(s)=K \frac{K_{d} s^{s}+s+K_{i}}{s}
$$

We see that $K$ and $K_{i}$ are of the same order of magnitude as the PI controller. As the controller becomes faster due to the derivative action, SS type-II errors are eliminated faster compared to the PI controller, for a reasonable small increase in overshoot.

The controller is implemented then in a microcontroller board which sends equivalent positive/negative values to the actuator. This is then evaluated in terms of zero-crossings (ZC) as an indicator of controller effort (aggressiveness). The actual control signals going into the process are therefore quite noisy, with very fast $\mathrm{ZC}$ frequencies and have been omitted from the paper.

\section{RESUlts}

The control performance is evaluated on a specially designed setup, for a ramp with slope $9^{\circ} / s$ slope and inputoutput slew rates as in section II. The lab device is depicted in Figure 5.

Three situations are envisaged:

- a perfectly known model (ideal situation);

- a drop in system gain of $-50 \%$ and

- an extra pole added to the system at $s=-10$.

The changes in gain and added dynamics reflect changes in the static characteristic and in the velocity components of the system under various operating conditions. Typical operating signals from application field have been used to test the controllers: step response, ramp and tracking signals. 

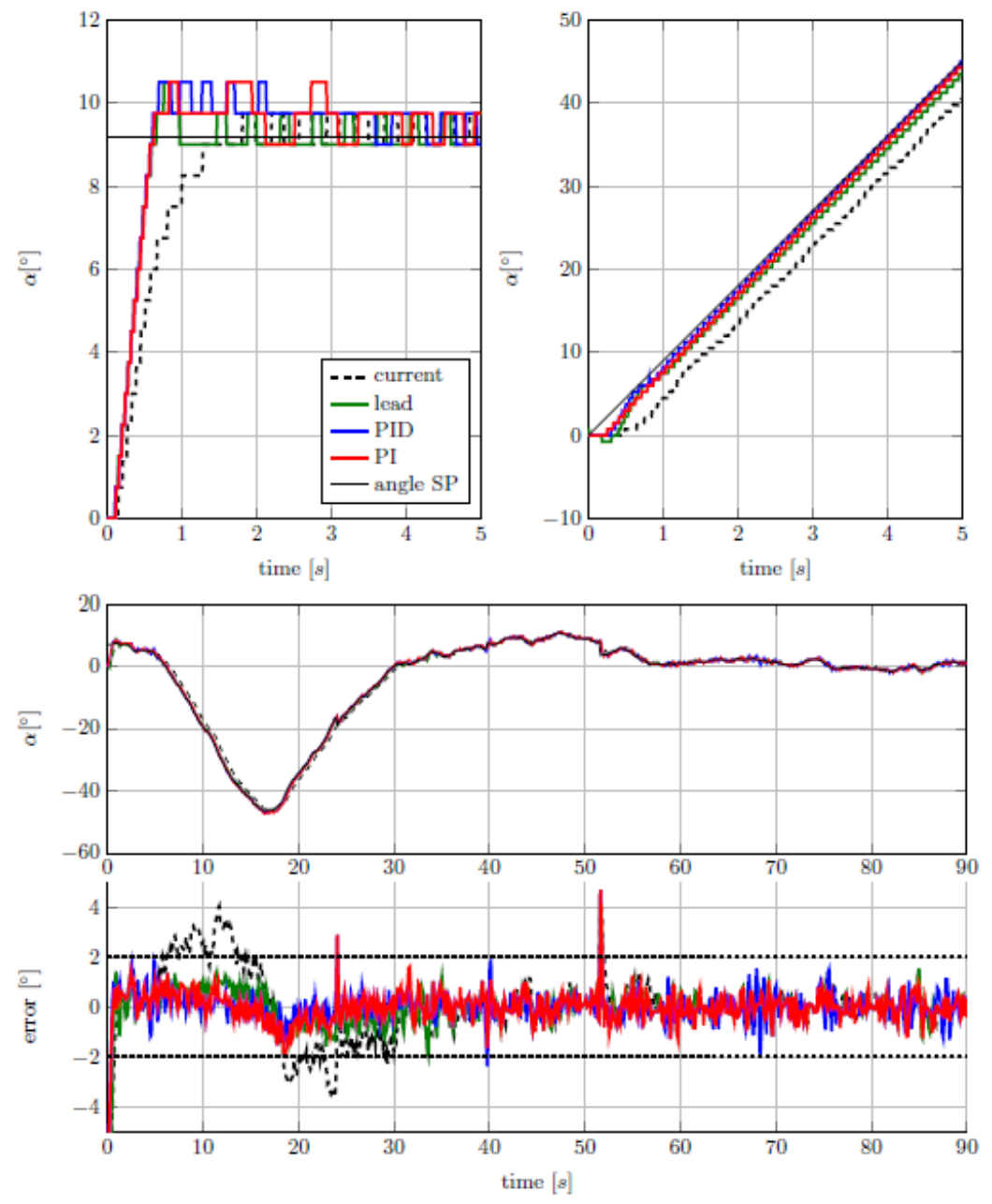

Fig. 6. Ideal situation: no modelling errors. Error tolerance between the two dashed lines.

The closed loop performance of the various controllers is given in figure 6 for the ideal case, in figure 7 for changes in the gain and in figure 8 for extra dynamics in the system. Performance in terms of integrated absolute error (IAE) and frequency of zero crossings in the controller output (ZC) are summarized in table I.

The default controller (in figure legend denoted by 'current') has good response for step reference but has a significant SS error for ramp input. This error increases with gain variations and with additional dynamics in the loop. It also cannot cope with the tolerance interval in the error values $( \pm 2$ degrees). The PI controller seems to have most overshoot for additional dynamics in the loop and relatively good results in all other situations. However, spikes in the error values are more frequent out of the tolerance interval for PI control than for other controllers.

The disadvantage of the D-action is however the number of ZC (zero crossings denote the change in direction), which is higher compared to other controller types. This is due to the low resolution sensor, as it renders an error signal which is very chattering. The signal requires a lot of filtering as
TABLE I

IAE IN DEGREES AND ZC IN FREQUENCY HZ

\begin{tabular}{cccc}
\hline & ideal case & gain $-50 \%$ & pole at $\mathrm{s}=-10$ \\
\hline default & $0.86 / 2.1$ & $1.48 / 1.1$ & $0.91 / 1.6$ \\
lead & $0.49 / 4.6$ & $0.67 / 2.7$ & $0.58 / 4.0$ \\
PID & $0.42 / 8.0$ & $0.44 / 5.2$ & $0.52 / 6.5$ \\
PI & $0.44 / 2.5$ & $0.57 / 1.5$ & $0.66 / 2.0$ \\
\hline
\end{tabular}

otherwise the controller output becomes very chattering too. Adding the derivative action to the feedback branch only, in order to cope with jumps in the input signal appearing quite often, is impossible in the current system setup. Hence, from this point of view, PID performs poorly. The PI controller shows best results on average.

Evaluating from the point of view of performance versus control effort (zero crossings), table II helps to make a decision. From this table, it seems that none of the controller types discussed in-so-far is able to satisfy all performance criteria simultaneously. We conclude therefore that more advanced strategies are necessary. 

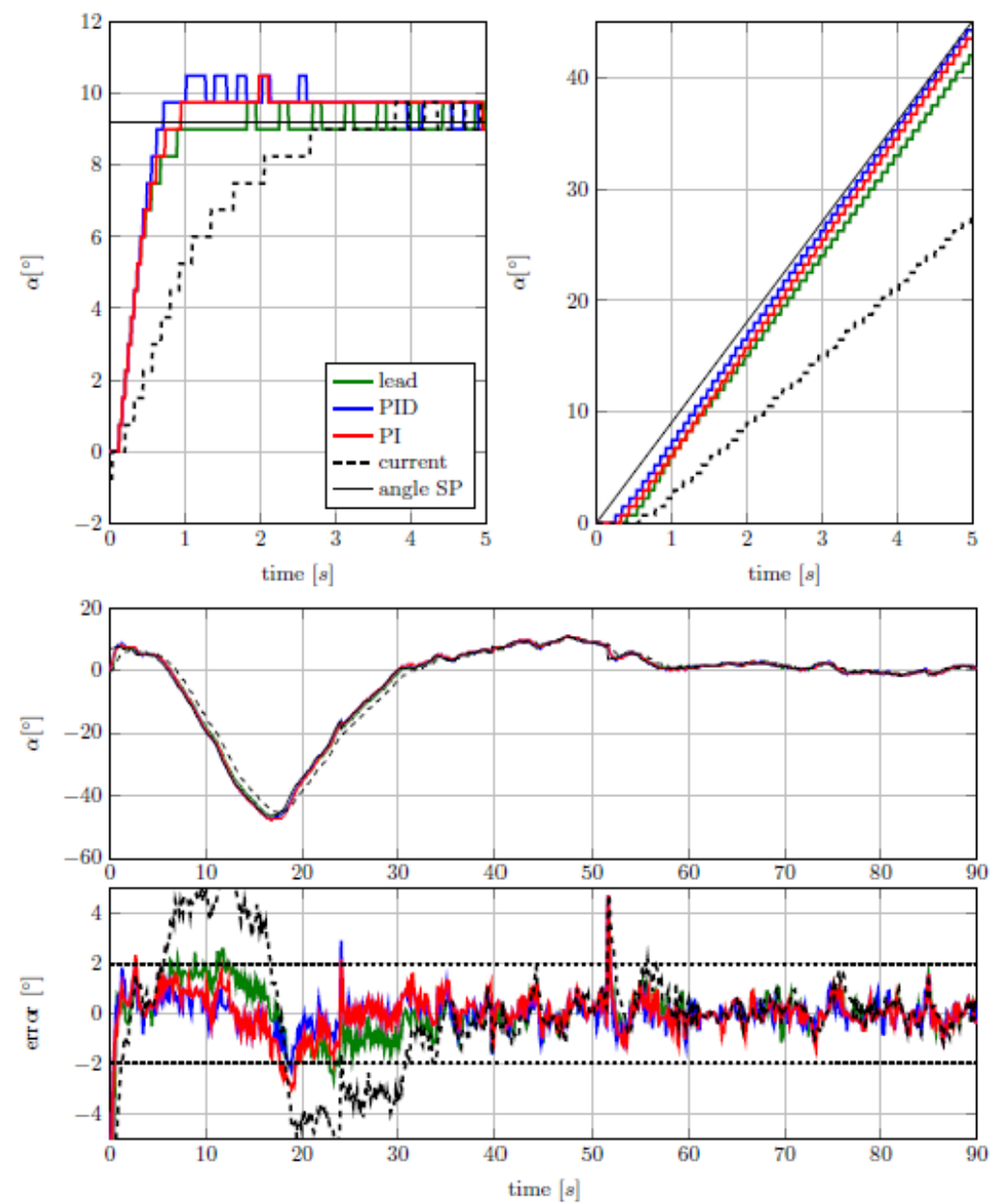

Fig. 7. Change in $50 \%$ gain situation. Error tolerance between the two dashed lines.

TABLE II

GLOBAL ASSESSMENT OF CLOSED LOOP PERFORMANCE

\begin{tabular}{ccccc}
\hline Controller & Error & SS-II & Robust & ZC \\
\hline default & + & - & \pm & + \\
Lead & \pm & \pm & - & \pm \\
PI & + & + & - & + \\
PID & + & + & + & - \\
\hline
\end{tabular}

\section{CONClusion}

This paper evaluated linear control strategies for a class of mechanical actuator systems commonly found in harvesting machines. Typically, these systems are classified as Hammerstein-type systems, with static nonlinear characteristics and fast dynamics. Additional dynamics as time-delays are introduced to mimic real life situations and closed loop performance is evaluated with slew rates on input-output variables.

Our results with simulated and real experimental data suggest that neither lead, P, PI nor PID control could satisfy global requirements as IAE and number of zero-crossings in the control effort (due to static nonlinearity). Current efforts are being made to test model based control strategies, as (adaptive) internal model control and sliding mode control on the real setup. This makes the scope of a future paper.

\section{REFERENCES}

[1] M. M. Schechter and M. B. Levin, "Camless engine" , SAE Technical Paper (1996), no. 960581

[2] A. di Gaeta, V. Giglio, and G. Police, "Model-based decoupling control of a magnet engine valve actuator", SAE International Journal of Engines, 2,pp. 254-271, 2010

[3] W. Saeys, B. Lenaerts, G. Craessaerts, J. De Baerdemaeker, "Estimation of the crop density of small grains using LiDAR sensors", BIOSYSTEMS ENGINEERING 102(1), pp. 22-30, 2009

[4] M. Reyniers, K. Maertens, E. Vrindts, J. De Baerdemaeker, "Yield variability related to landscape properties of a loamy soil in central Belgium", SOIL \& TILLAGE RESEARCH, 88(1-2), pp. 262-273, 2006

[5] B. Lenaerts, T. Aertsen, E. Tijskens, B. De Ketelaere,H. Ramon, J. De Baerdemaeker, W. Saeys, "Simulation of grain-straw separation by Discrete Element Modeling with bendable straw particles", COMPUTERS AND ELECTRONICS IN AGRICULTURE, 101, pp. 24-33, 2014

[6] R. Poley, "DSP control of electro-hydraulic servo actuators", Texas Instruments, Tech. Rep., 2005.

[7] D. Maneetham, N. Afzulpurkar, "Modeling, simulation and control of high speed nonlinear hydraulic servo system", World Journal of Modelling and Simulation,6(1), pp. 27-39, 2010. 

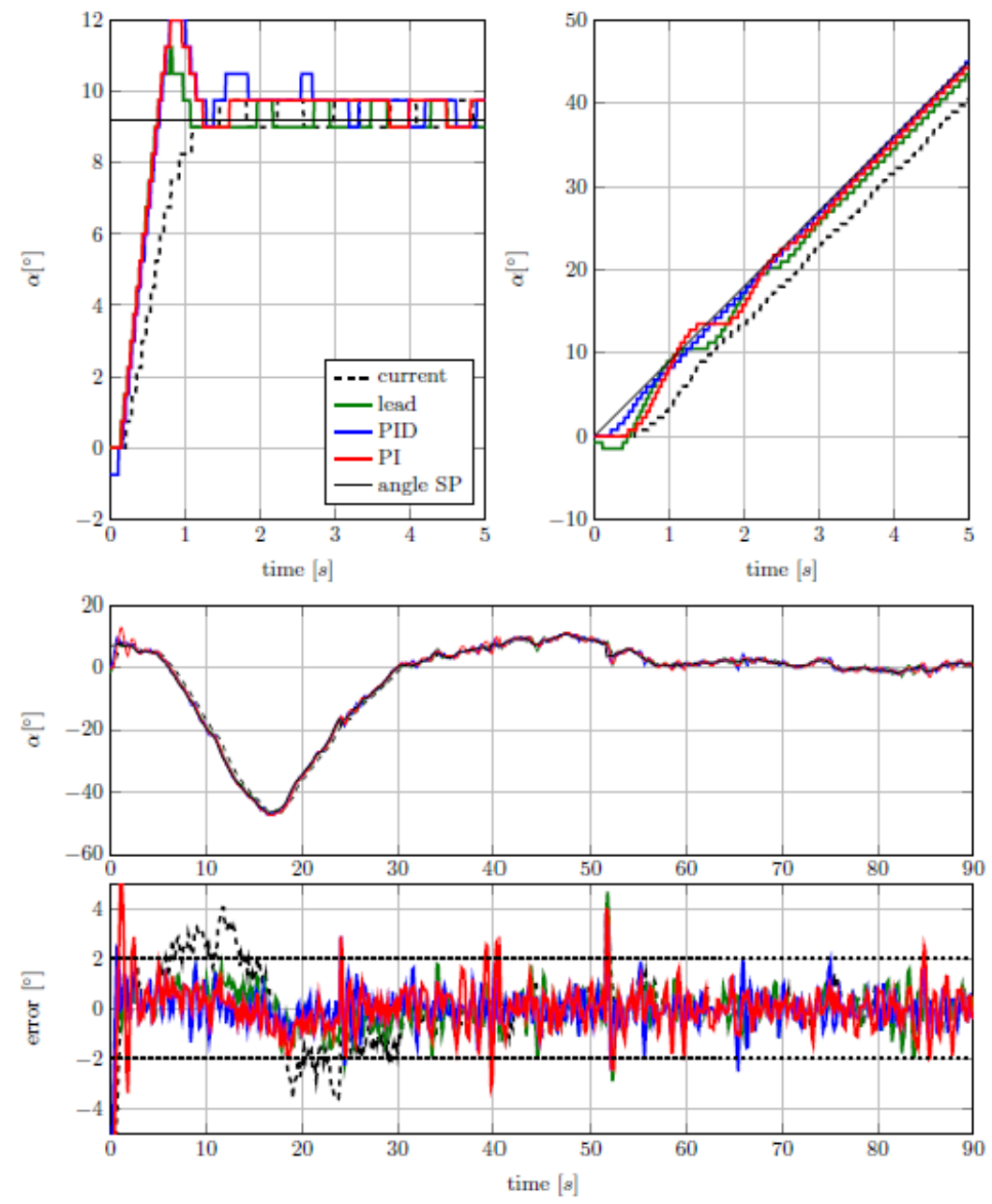

Fig. 8. Additional dynamics situation. Error tolerance between the two dashed lines.

[8] B. Yao et al., "Adaptive robust motion control of single-rod hydraulic actuators: theory and experiments", IEEE/ASME Transactions on Mechatronics,5(1), pp. 79-91, 2000.

[9] H. Merrit, Hydraulic Control System. John Wiley \& Son, inc., 1967.

[10] K. Dasgupta, H. Murrenhofi, "Modelling and dynamics of a servo-valve controlled hydraulic motor by bondgraph", Mechanism and Machine Theory, 46, pp. 1016-1035, 2011.

[11] J. Sjoberg et al., "Nonlinear black-box modeling in system identification: a unified overview", Automatica, 31(12), pp. 1691-1724, 1995.

[12] I. Rivals , L. Personnaz, "Black-box modelling with state-space neural networks", Neural Adaptive Control Technology, pp. 237-264, 1996.

[13] C.M. Ionescu, R. Hodrea, R. De Keyser, "Variable Time-Delay Estimation for Anesthesia Control During Intensive Care", IEEE TRANSACTIONS ON BIOMEDICAL ENGINEERING, 58(2), pp. 363-369, 2011

[14] C. Pop, C.M. Ionescu, R. De Keyser, E.Dulf, " Robustness evaluation of fractional order control for varying time delay processes", SIGNAL IMAGE AND VIDEO PROCESSING, 6(3), Special Issue: SI, pp. 453461,2012

[15] C. Pop, C.M. Ionescu, R. De Keyser, " Time delay compensation for the secondary processes in a multivariable carbon isotope separation unit", CHEMICAL ENGINEERING SCIENCE, 80, pp. 205-218, 2012

[16] M. Galvez-Carrillo, R. De Keyser, C.M. Ionescu, "Nonlinear predictive control with dead-time compensator: Application to a solar power plant", SOLAR ENERGY, 83(5), pp. 743-752, 2009

[17] J. Nino, R. De Keyser, S. Syafiie, C.Ionescu, M. Struys, "EPSAC controlled anesthesia with online gain adaptation", INTERNATIONAL JOURNAL OF ADAPTIVE CONTROL AND SIGNAL PROCESSING, 23(5), Special Issue: SI, pp. 455-471, 2009
[18] R. De Keyser, C.M. Ionescu, "FRtool: a frequency response tool for CACSD in Matlab", IEEE Conf. on Computer Aided Control Systems Design, Munich, pp. 2275-2280, 2006. 